\title{
A Survey of Mobile Ad Hoc Reactive Routing Protocols in Two Different Mobility Models
}

\author{
M.K.Jeya Kumar and R.S.Rajesh
}

\begin{abstract}
Ad Hoc Networks is a multi - hop wireless network with dynamically changing network connectivity owing to mobility. It consists of a collection of wireless mobile nodes it will act without the use of any existing infrastructure or centralized administration. In this dynamic network, routing is a challenging problem Different routing protocols are designed assuming certain mobility patterns of the mobile nodes in the network. However the choice of mobility pattern may favor certain protocols over the others. Hence it is necessary to study the performance of different routing protocols under different mobility models. In this work the two mobility models Probabilistic Random Walk and Boundless Simulation Area with high mobility and Low mobility constraints have been considered. The two reactive protocols AODV and DSR are examined based on the traces derived for each of these mobility models for various mobility speed, Traffic and Node Density in the network. The simulation result shows that, in probabilistic Random walk model, AODV yields good performance for high/low mobility, high/low traffic and sparse/dense networks. In Boundless simulation area model the performance produced by both the protocols are almost same.
\end{abstract}

Index Terms-Ad Hoc Networks, Mobility Models, AODV, DSR, Routing Protocols

\section{INTRODUCTION}

A mobile ad hoc network (MANET) is an autonomous system of mobile hosts connected by wireless links. There is no static infrastructure such as base stations. Each node in the network also acts as a router, forwarding data packets to other nodes. Any number of people could conceivably enter a conference room and agree to support communication links[6] between themselves, without necessarily engaging the services of any pre-existing equipment in the room. Thus, it is a temporary network with no wires and no administration intervention required.

A central challenge in the design of ad hoc networks is the development of dynamic routing protocols[6] that can efficiently find routes between two communicating nodes. The routing protocols[5] must be able to cope up with the high degree of node mobility[11] that often changes the network topology drastically and unpredictably.

The various ad hoc routing protocols have their unique

M.K.Jeya Kumar is a Research Scholar of Dr.M.G.R.University, Tamilnadu, Chennai., India. (Ph.No. 9443281067)

Dr.R.S.Rajesh is with Department of Computer Science \& Engineering., Manonmaniam Sundaranar University, Tirunelveli, Tamilnadu, India. (Ph.No. 9443869904) characteristics. Hence, in order to find out the most adaptive and efficient routing protocol for the highly dynamic topology in ad hoc networks, the routing protocols behavior has to be analyzed using varying node mobility speed, Network Traffic and Node Density. Thus, the goal is to carry out a systematic performance comparison of ad hoc routing protocols under mobility models.

The main aim of this paper is:

Acquiring the detailed understanding of ad hoc routing protocols such as AODV and DSR

Implementing the Mobility models such as Probabilistic Random Walk and Boundless simulation Area

Analyzing the performance differentials of routing protocols under mobility.

For implementing the mobility models and Routing Protocols Network Simulator (NS) has been used. NS is an object-oriented, discrete event driven network simulator developed at Barkely written in $\mathrm{C}++$ and OTcl. NS is object - oriented $\mathrm{Tcl}(\mathrm{OTcl})$ script interpreter that has simulation event scheduler and network component object libraries.

The organization of the paper is as follows. Section 2 discusses the major mobile Ad hoc routing protocols used in this evaluation study. Section 3 presents the mobility models used in this analysis. The simulation results, followed by their interpretations are presented in section 4. The concluding remarks are included in the 5 .

\section{MOBILE AD-HOC NETWORKING PROTOCOLS}

The main problem with ad-hoc networking is how to send a message from one node to another with no direct link. The nodes in the network are moving around unpredictably, and it is very challenging which nodes that are directly linked together.. The topology of an ad-hoc network is constantly changing and it is very difficult for routing process. There are two main approaches for routing process in ad hoc networks. The first approach is a pro-active approach which is table driven and uses periodic protocols. This means that all nodes have tables with routing information which are updated at intervals. The second approach is re-active, source-initiated or on-demand. This means that every time a message is sent it first has to find a path by searching the entire network. There are many different protocols that are in accordance with the two different routing approaches. Different protocols are specialized in different aspects of the routing such as finding a short path, low overhead communication and load-balancing.

The AODV and DSR are source-initiated or on-demand routing protocols[13]. The two ad hoc routing protocols 
considered in this study are explained below.

\section{A. Ad-Hoc On Demand Distance Vector Routing- $A O D V$}

The Ad-hoc On-demand Distance Vector routing protocol [1][7] enables multihop routing between the participating mobile nodes wishing to establish and maintain an ad-hoc network. AODV is a reactive protocol based upon the distance vector algorithm[3].

The algorithm uses different messages to discover and maintain links. Whenever a node wants to try and find a route to another node, it broadcasts a Route Request (RREQ) to all it's neighbors. The RREQ propagates through the network until it reaches the destination or the node with a fresh enough route to the destination. Then the route is made available by uncasing a RREP back to the source.

The algorithm uses hello messages (a special RREP) that are broadcasted periodically to the immediate neighbors. These hello messages are local advertisements for the continued presence of the node, and neighbors using routes through the broadcasting node will continue to mark the routes as valid. If hello messages stop coming from a particular node, the neighbor can assume that the node has moved away and mark that link to the node as broken and notify the affected set of nodes by sending a link failure notification (a special RREP) to that set of nodes.

\section{B. Dynamic Source Routing-DSR}

The Dynamic Source Routing (DSR) [4] is a reactive unicast routing protocol that utilizes source routing algorithm. In source routing algorithm, each data packet contains complete routing information to reach its dissemination. Additionally, in DSR each node uses caching technology to maintain route information that it has learnt.

There are two major phases in DSR, the route discovery phase and the route maintenance phase. When a source node wants to send a packet, it firstly consults its route cache. If the required route is available, the source node includes the routing information inside the data packet before sending it. Otherwise, the source node initiates a route discovery operation by broadcasting route request packets. A route request packet contains addresses of both the source and the destination and a unique number to identify the request. Receiving a route request packet, a node checks its route cache. If the node doesn't have routing information for the requested destination, it appends its own address to the route record field of the route request packet. Then, the request packet is forwarded to its neighbors. If the route request packet reaches the destination or an intermediate node has routing information to the destination, a route reply packet is generated. When the route reply packet is generated by the destination, it comprises addresses of nodes that have been traversed by the route request packet. Otherwise, the route reply packet comprises the addresses of nodes the route request packet has traversed concatenated with the route in the intermediate node's route cache.

In DSR, when the data link layer detects a link disconnection, a ROUTE_ERROR packet is sent backward to the source. After receiving the ROUTE_ERROR packet, the source node initiates another route discovery operation. Additionally, all routes containing the broken link should be removed from the route caches of the immediate nodes when the ROUTE_ERROR packet is transmitted to the source.

DSR has increased traffic overhead by containing complete routing information into each data packet, which degrades its routing performance.

\section{MOBILITY MODELS}

The mobility models considered in this evaluation is Probabilistic Random Walk and Boundless Simulation Area are explained below.

\section{A. Probabilistic Random Walk}

Chiang's mobility mode[12] utilizes a probability matrix to determine the position of a particular $\mathrm{MN}$ in the next time step, which is represented by three different states. State 0 represents the current location of given $\mathrm{MN}$, state 1 represents the MN's next position, and state 2 represents the MN's next location if the MN moves forward. The probability matrix used is

$$
\mathrm{P}=\left[\begin{array}{lll}
\mathrm{P}(0,0) & \mathrm{P}(0,1) & \mathrm{P}(0,2) \\
\mathrm{P}(1,0) & \mathrm{P}(1,2) & \mathrm{P}(1,2) \\
\mathrm{P}(2,0) & \mathrm{P}(2,1) & \mathrm{P}(2,2)
\end{array}\right]
$$

where each entry $\mathrm{P}(\mathrm{a}, \mathrm{b})$ represents the probability that an $\mathrm{MN}$ will go from state a to state $\mathrm{b}$. Here state 0 denotes the current location, state 1 denotes the previous location and state 2 denotes the next location.

The mobility patterns for the mobility of nodes with probability matrix $\mathrm{P}$ is given here. For each chosen probability value the node travels for a distance of 10 meters with a constant velocity of $10 \mathrm{~m} / \mathrm{s}$

$$
\mathrm{P}=\left[\begin{array}{lll}
0.0 & 0.5 & 0.5 \\
0.3 & 0.7 & 0.0 \\
0.3 & 0.0 & 0.7
\end{array}\right]
$$

In this model, the distribution of the nodes does not change heavily and also the average number of neighbors is static. This will keep the number of hops be minimum, so it reduces the end-to-end delay in delivery the packets.

\section{B. Boundless Simulation Area}

In the Boundless Simulation Area Mobility Model, a relationship between the previous direction of travel and velocity of an $\mathrm{MN}$ with its current direction of travel and velocity exists [9]. A velocity vector $\bar{v}=(v, \theta)$ is used to describe an MN's velocity $v$ as well as its direction $\theta$; the MN's position is represented as $(x ; y)$. Both the velocity vector and the position are updated at every $\Delta t$ time steps according to the following formulas:

$$
\begin{aligned}
& v(t+\Delta t)=\min [\max (v(t)+\Delta v, 0), V \max ] ; \\
& \theta(t+\Delta t)=\theta(t)+\Delta \theta ; \\
& x(t+\Delta t)=x(t)+v(t) * \cos \theta(t) ; \\
& y(t+\Delta t)=y(t)+v(t) * \sin \theta(t) ;
\end{aligned}
$$


where $V_{\max }$ is the maximum velocity defined in the simulation, $\Delta_{v}$ is the change in velocity which is uniformly distributed between $\left[-A_{\max } * \Delta t, A_{\max } * \Delta t\right], A_{\max }$ is the maximum acceleration of a given $\mathrm{MN}, \Delta \theta$ is the change in direction which is uniformly distributed between $[-\alpha * \Delta t, \alpha * \Delta t]$, and $\alpha$ is the maximum angular change in the direction an $\mathrm{MN}$ is traveling.

The Boundless Simulation Area Mobility Model is also different in how the boundary of a simulation area is handled. In this mobility Model, MNs that reach one side of the simulation area continue traveling and reappear on the opposite side of the simulation area. When the node encounters the simulation boundary, it appears on the opposite side of the simulation area and continues traveling at the same angle and velocity. When $\Delta t$ time steps finish, the $\mathrm{MN}$ chooses a new direction and velocity evaluated based on the previous direction and velocity and begins traveling again In effect, this technique creates a torus-shaped simulation area that are allowing MNs to travel unobstructed. The node reaching the boundary reappears on the opposite side of the simulation area. When it reappears, the MNs parameters like the speed, direction are not changed and also the MNs does not stop there. This will generate mobility without any sharp turns or sudden increase/decrease of velocity of the MN. Also the distribution will be uniform which will ensure constant number of neighbors for all the MNs.

\section{PERFORMANCE RESULTS}

This section discusses the various predominance metrics used and the Performance differentials analyzed[8]. The performance metrics analyzed are the fraction of packets delivered at the destination and the packet delivery ratio for various speeds of mobility, Traffic and Network Size.

The simulation is done with different nodes in wireless sensor networks with respect to the Probabilistic random walk mobility model and Boundless Simulation Area mobility models. The protocols considered for analysis are AODV and DSR.

\section{A. Speed vs Packet Delivery Fraction}

The Performance of the routing protocols in terms of packet delivery ratio is examined with respect to the mobility of nodes[6]. Tow different network traffic density scenarios[2] are considered one with 10 connections and another with 20 connections. The simulation results are shown in the fig. 1 .

The differences in packet delivery ratios produced by probabilistic Random Walk and Boundless simulation area are very less. The result produced by AODV is almost stable for low and high mobility. In probabilistic Random Walk and Boundless simulation area, AODV Protocols perform better than DSR in lower and higher mobility status.

\section{B. B Speed vs End-to-End delay}

The performance of the routing protocols in terms of End-to-End Delay is examined with respect to mobility of the nodes. End-to-end delay are considered for 10 connections and 20 connections traffic density. The results are shown in the fig. 2 .
The end-to-end delay difference in Probabilistic Random Walk and Boundless Simulation Area is very less. The AODV in probabilistic Random walk model perform best in low mobility and high mobility conditions. In Boundless simulation Area, DSR perform better than AODV in low mobility and AODV performs best in higher mobility status.

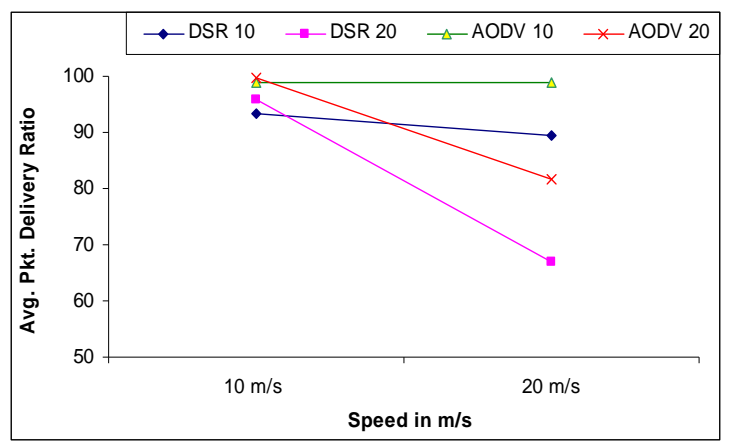

(a) Probabilistic Random Walk

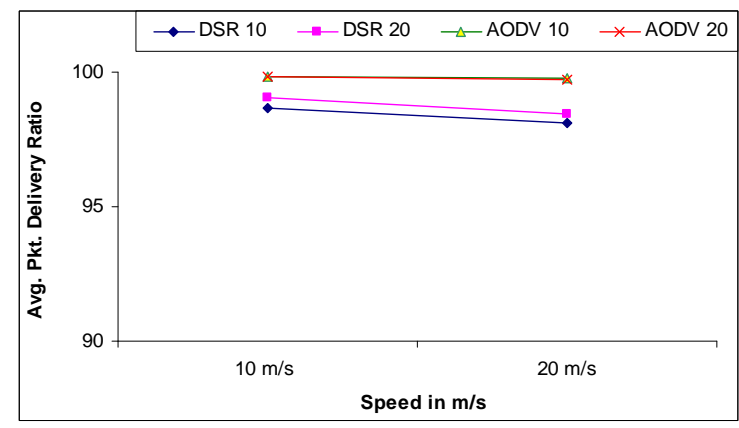

(b) Boundless Simulation Area

Figure 1. Packet Delivery Fraction for varying speeds

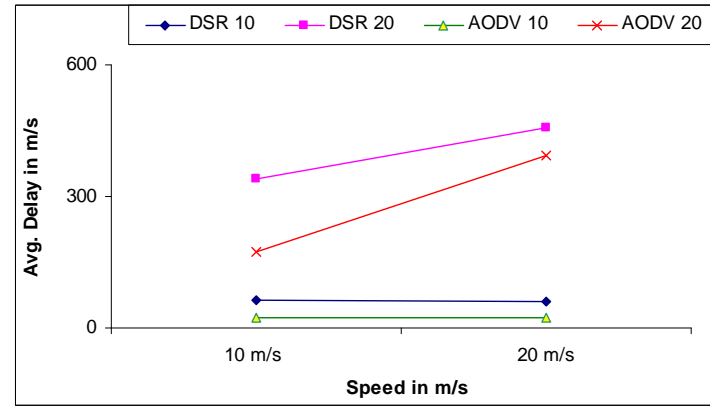

(a) Probabilistic Random Walk

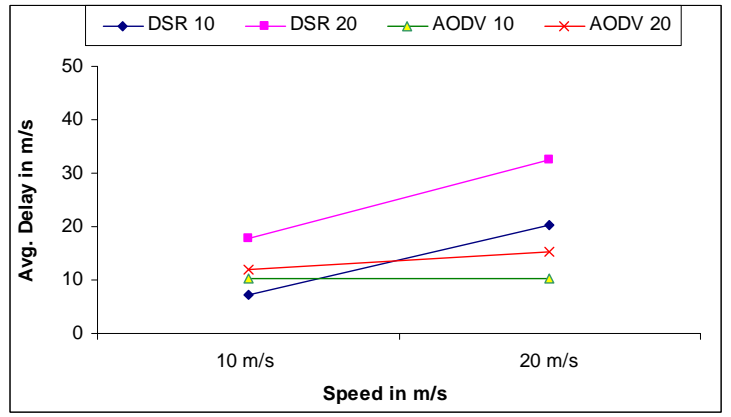

(b) Boundless Simulation Area

Figure 2. Speed vs End-to-end Delay 


\section{Traffic vs Packet Delivery Ratio}

The performance of the routing protocols in terms of packet delivery ratio is examined with respect to traffic load. Tow different network traffic density scenarios are considered one with 10 connections and another with 20 connections. The simulation results are shown in the fig. 3 .

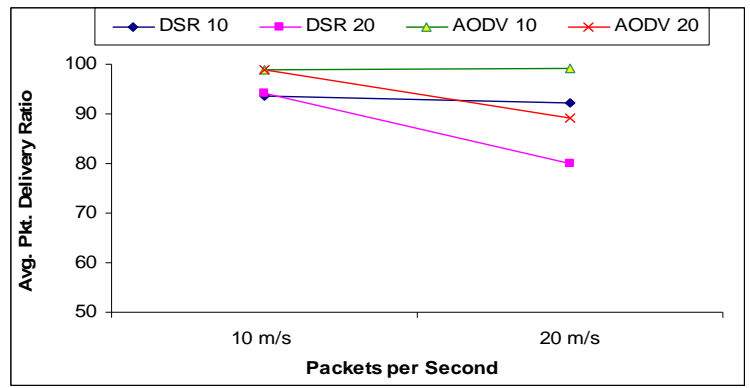

(a) Probabilistic Random Walk

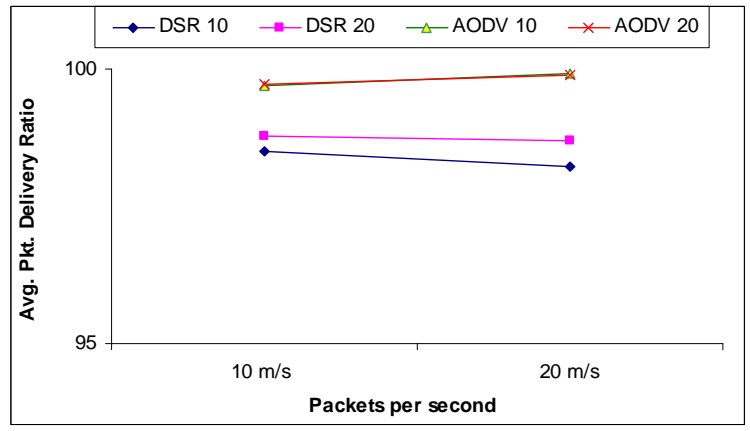

(b) Boundless Simulation Area

Figure 3. Traffic vs Packet Delivery Ratio

The packet delivery ratio obtained from the simulation show sharp decrease when the number of packets is increased from 1 to 4 and number of connections is increased from 10 to 20. In Probabilistic Random Walk Model, there is a slight difference in packet delivery ratio, where it is static in Boundless simulation area mobility model. AODV Protocol performs better than DSR in low traffic and high traffic conditions.

\section{Traffic vs End-to-End Delay}

The performance of the routing protocols in terms of End-to-End Delay is examined with respect to traffic load. End-to-end delay are considered for 10 connections and 20 connections traffic density. The simulation results are shown in the fig. 4.

In both the mobility models the routing protocols consume less time to deliver packets with 10 connections and 1 packet per second/connections protocols. More time is spending to deliver packets when the number of packets and connections are increased. The difference in the time spends by both the protocols under probabilistic Random Walk and Boundless Simulation Area is very less. AODV Performs better than DSR in Probabilistic Random Walk model in low and high traffic. In low traffic condition AODV performs best in Boundless Simulation Area and DSR performs best in higher traffic conditions.

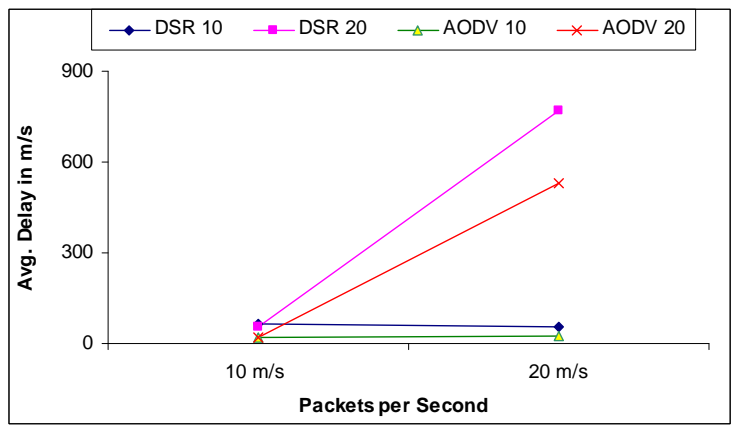

(a) Probabilistic Random Walk

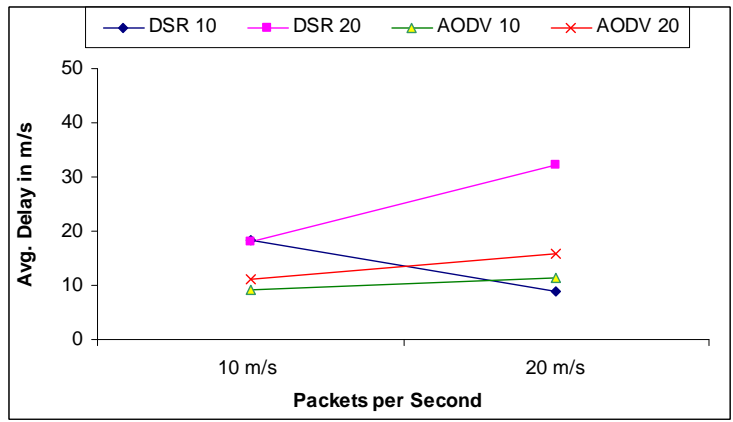

(b) Boundless Simulation Area

Figure 4. Traffic vs End-to- Delay

\section{E. Node density Vs Packet Delivery Fraction}

The performance of the Routing protocols in terms of packet delivery ratio is examined with respect to the area in which the nodes are likely to move. Packet delivery ratios are considered for 10 connections and 20 connections traffic density. The simulation results are shown in the fig. 5 .

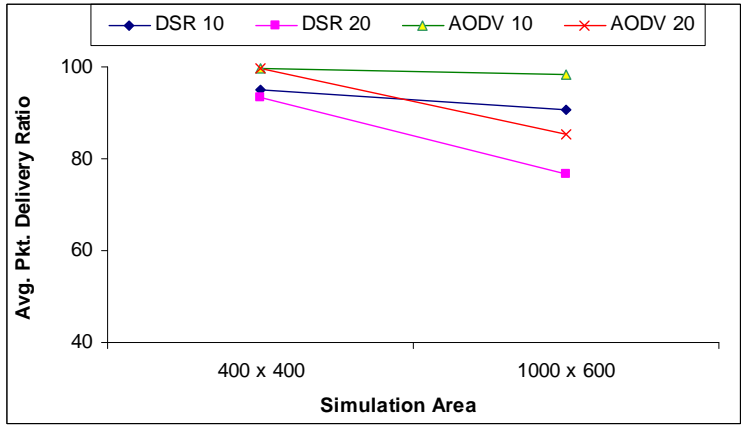

(a) Probabilistic Random Walk

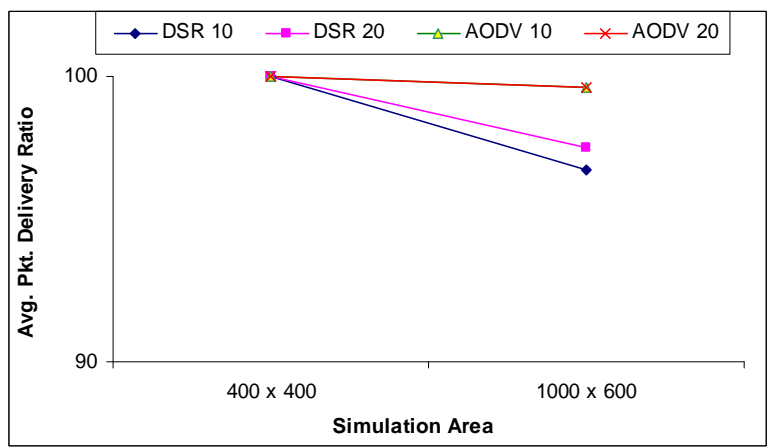

(b) Boundless Simulation Area

Figure 5. Node Density vs Packet Delivery Ratio 
In Probabilistic random Walk and Boundless Simulation area, the packets delivery ratio generated by both the protocols do not show any considerable difference. In both the mobility models AODV Protocols performs best in lower and higher Node Density status.

\section{F. Node Density vs End-to-End Delay}

The performance of the routing protocols in terms of end-to-end delay is examined with respect to the area with in which the nodes are likely to move. Two traffic density scenarios are considered, one with 10 connections and another with 20 connections. The results are shown graphically in fig. 6 .

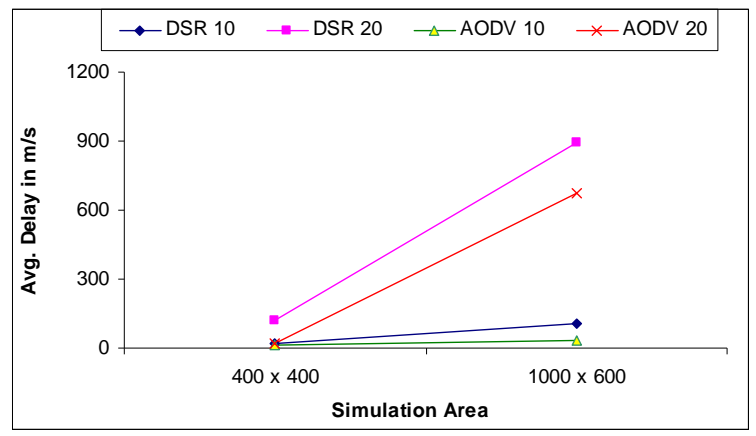

(a) Probabilistic Random Walk

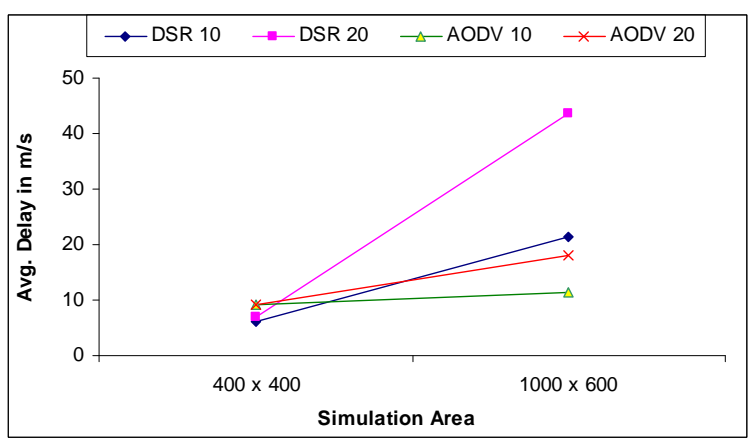

(b) Boundless Simulation Area

Figure 6. Node Density vs End-to-End Delay

The end-to-end delay is very less with higher node density and increases heavily when the node becomes sparse. The delay time consumed by DSR in Boundless Simulation Area is lesser than AODV in high and slightly higher in lesser node density.

\section{DISCUSSION}

In probabilistic Random Walk model does not show any sharp turn and sudden stops. The number of neighbors becomes average and hop distance is less. This reduces the delay and increases Packet delivery Ratio. So the AODV protocols provide better results than DSR.

In the Boundless simulation Area model the node is traveling unobstructed throughout the entire simulation area and it avoids the edge effects caused in all the remaining models. The traveling pattern of the mobile nodes is smoother and speed and direction to travel in each step depends on the previous speed and direction. So both the protocols provide best result.

\section{CONCLUSION}

The two mobility models, Probabilistic Random Walk and Boundless Simulation Area mobility models and the two familiar Ad Hoc routing protocols AODV and DSR have been implemented and analyzed. The Packet Delivery Ratio and End-to-End Delays of the Routing protocols AODV and DSR has been evaluated with respect to the parameters such as mobility speed, network traffic and node density.

In the probabilistic Random walk model, AODV yields good performance for high/low mobility, high/low traffic and sparse/dense network. But the performance of DSR is good for low traffic and low mobility. In Boundless simulation area model the performance produced by both the protocols are almost same, but AODV may be advisable for sparse distribution.

The main future enhancement of this paper is as follows. In this paper only considered the number of nodes as constant throughout the simulation. But this could be varied dynamically so as to make the network becomes a scalable network and thus the network becomes scalable. The various parameters used for propagation model, Link layer, MAC Layer, Interface Queue and the antenna type in the Mobile Ad Hoc nodes can also be modified so as to evaluate more practical mobile networks.

\section{REFERENCES}

[1]. Amir R.Das, Charles E.Perkins and Elizabeth M.Royer. "An Implementation Study of the AODV routing protocol", Proceedings of the IEEE Wireless Communication and Networking Conference (INFOCOM), Tel Aviv, Isrel, 2000, pp 3-12.

[2]. B.L. Agba, F. Gagnon, A. Kouki, "Scenarios generator for ad hoc networks" International Symposium on Industrial Electronics, Montreal, Canada, 2006, 2677-2681

[3]. C. Perkins and E. Royer. "Ad Hoc On-Demand Distance Vector Routing". The $2^{\text {nd }}$ IEEE workshop on Mobile Computing Systems and Applications(WMCSA '99), New Orleans, 1999, pp 90-100.

[4]. Jochen Schiller "Mobile Communications", Addision Wesley Longman Pvt.Ltd, India. 2000.

[5]. Josh Broch, David A.Maltz, David B. Johnson Yih-Chen Hu and Jorjeta Jetcheva, "A Performance Comparison of Multihop Wireless Ad Hoc Network Routing Protocols", ACM MOBICOM '98, Dallas, Texas. pp 25-30, 1998.

[6]. P. Johanson, T. Larsson, N. Hedman, Mielczarek and M.Degrermark., "Routing Protocols for Mobile Ad Hoc Networks - a comparative performance analysis", Proceedings of the ACM/IEEE International Conference on Mobile Computing and Networking(MOBICOM), Seattle, WA, 1999, pp 195-206.

[7]. Samir. R. Das, R. Castaneda and J.Yan. "Simulation based Performance Evaluation of Routing Protocols for Mobile Ad-hoc Networks"ACM/Baltzer Mobile Networks and Applications (MONET), pp 179-189, 2000.

[8]. Samir R. Das, Robert Castaneda, Jiangtao Yan, Rimli Sengupta, "Comparative Performance Evaluation of Routing Protocols for Mobile Ad Hoc Networks", $7^{\text {th }}$ International Conference on on Computer Communication and networks( IC3N, pp 153-161, 1998.

[9]. The VINT Project, The Network Simulator - ns-2, URL: http://www.isi.edu/nsnam/ns/. Page accessed on April 12th, 2003.

[10]. Tony Larsson, Nicklar Hedman, "Routing Protocols in Wireless Ad Hoc Networks - A Simulation Study", Masters thesis in Computer Science and Engineering, Stockholm, Lulea University of Technology, 1998.

[11]. Tracy Camp, Jeff Boleng, Vanessa Davies, "A Survey of Mobility Models in Wireless Ad-hoc Networks" Wireless Communications \& Mobile Computing (WCMC): Special issue on Mobile Ad Hoc Networking: Research, Trends and Applications, vol. 2, no. 5, pp. 483-502, 2002.

[12]. Vanessa Ann Davies, "A Thesis on "Evaluating Mobility Models within an ad-hoc networks", Colaroda School of Mines, 2000. 
[13]. Z. Haas, . A new routing protocol for reconfigurable wireless networks. In Proceedings of the IEEE International Conference on Universal Personal Communications (ICUPC), CA, USA. 1997, Pp562-565.

M.K.Jeya Kumar received his MCA degree from Bharathidasan University, Trichirappalli in the year 1993. He fetched his M.Tech. degree from Manonmaniam Sundaranar University, Tirunelveli in the year 2005. His area of interests are Computer Networks and Sensor Networks. He is currently pursuing his Ph.D degree in Mobile Ad Hoc Network in Dr.M.G.R University, Chennai under the guidance of Dr. R.S.Rajesh. Presently he is working as a Faculty in the Department of Computer Applications, Noorul Islam University, Kumaracoil, India since 1994.

Dr. R. S Rajesh received his B.E and M.E degrees in Electronics and Communication Engineering from Madurai Kamaraj University, Madurai, India in the year 1988 and 1989 respectively, and completed his Ph.D in Computer Science and Engineering from Manonmaniam Sundaranar University in the year 2004. In September 1992 he joined in Manonmaniam Sundaranar University where he is currently working as Professor in the Department of Computer Science and Engineering. He is having more than 19 years of PG teaching and Research experience. His current research interests include, Wireless networks, Pervasive computing, Digital image processing and parallel Computing. 